\title{
CARACTERIZACIÓN DE FINCAS AGROPECUARIAS DE EL TINGO LA ESPERANZA / PUJILÍ / COTOPAXI / ECUADOR
}

\section{CHARACTERIZATION OF AGRICULTURAL FARMS FROM EL TINGO LA ESPERANZA / PUJILI / COTOPAXI / ECUADOR}

\author{
Emerson Jácome Mogro ${ }^{1,2}$, Alexander Rodríguez-Berrío ${ }^{3}$, Santiago Jiménez \\ Jácome $^{1,4}$, Karina Marín Quevedo ${ }^{1,5}$ y Vinicio Mogro Cepeda ${ }^{1,6}$
}

\begin{abstract}
Resumen
El trabajo se realizó en la Parroquia El Tingo La Esperanza del Cantón Pujilí, Provincia de Cotopaxi, en la parte occidental de la Cordillera de los Andes Centrales de Ecuador, con el objetivo de caracterizar a los productores agropecuarios del sector. Se trabajó con los siete principales asentamientos humanos dando una muestra de 109 unidades productivas agropecuarias (UPA) de 844 productores registrados. Los resultados indicaron que los sistemas agropecuarios en el sector son diversos, donde el mayor número de familias se dedican al engorde de ganado vacuno, el cultivo de caña y el cultivo de mora; con pocas áreas dedicadas a la diversificación de cultivos, con limitado uso de tecnología cuya producción es destinada al autoconsumo. Se evidenció que los servicios básicos en la zona de estudio son deficientes. El análisis de conglomerados indicó dos grupos de productores en función de sus similitudes, siendo los habitantes más vulnerables por la falta de vías de comunicación aquellos que están en las localidades de Siete Ríos y Yungañan. El otro agrupamiento estuvo conformado por Macuchi, El Tingo La Esperanza, El Palmar, Puembo y Choasilli.
\end{abstract}

Palabras clave: Andes Centrales, Ecuador, caracterizar, productores agropecuarios.

\begin{abstract}
The work was carried out at El Tingo La Esperanza Parish, Pujilí canton, Cotopaxi province, in the western part of the central Andes of Ecuador, with the objective of characterizing the agricultural producers in the sector. We worked with the seven main human settlements, in a sample of 109 agricultural production units (UPA) from 844 registered producers. The results indicate that the agricultural systems in the sector are diverse, where the largest number of families are dedicated to fattening cattle, sugar cane and blackberry farming; with few areas dedicated to crop diversification, with limited use of technology, the production of which is destined for self-consumption. It was evident that the basic services in the study area are deficient. The cluster analysis indicates two groups of producers based on their similarities, the most vulnerable population due to the lack of communication channels were the towns of: Siete Ríos and Yungañan. The other group was made up of: Macuchi, El Tingo La Esperanza, El Palmar, Puembo and Choasilli.
\end{abstract}

Key words: Central Andes, Ecuador, characterize, agricultural producers.

\section{Introducción}

Los países en vías de desarrollo enfrentan problemas propios de sus economías dependientes, obligándolos a aplicar sistemas agropecuarios tradicionales, los mismos que no son cuidadosos del ambiente, ni sustentables, ni rentables. Esto se puede evidenciar en la debacle agropecuaria, por los altos niveles de contaminación del ambiente, por tal razón, se genera la importancia de levantar la línea base de los habitantes del sector (Zayas, 2013).

En el Ecuador las estribaciones occidentales de los Andes han sido deforestadas continuamente, siendo la principal causa de la degradación de los ecosistemas, esta intervención antrópica en muchas ocasiones ha sido auspiciada por políticas estatales o por omisiones en el control del territorio, donde el bosque primario en un inicio fue desplazado por pasturas para engorde de ganado vacuno, además el sector fue afectado por minería aurífera, actualmente existe monocultivo de mora y caña, lo cual ocasiona pérdida de servicios ecosistémicos y consecuentemente bajos indicadores productivos de las actividades agropecuarias implementadas. En el centro de la problemática se encuentran los productores como responsables de la degradación ambiental. Sin embargo, es necesario generar un diagnóstico que promueva alternativas de cambio, en el que participen sectores académicos, representantes de los productores y las entidades estatales, para formular políticas sostenibles de cambio (Ramírez et al., 2012).

Según Sarandón et al. (2006), se han logrado avances importantes en la caracterización y evaluación 
de sustentabilidad de las fincas de los productores agropecuarios, pero aún persisten dificultades en la toma de decisiones de los aspectos holístico y sistémico, donde predomine el análisis multicriterio para lograr la sustentabilidad anhelada.

La investigación se apoyó en la generación de información de acuerdo con Bolaños (1999), a través de la toma de datos que permiten identificar las características de las unidades productivas agropecuarias (UPA).

En función del contexto indicado, esta investigación tuvo como objetivo caracterizar y tipificar las fincas de los sistemas agropecuarios prevalentes e indicar las limitantes de los productores de la Parroquia El Tingo La Esperanza.

\section{Materiales y Métodos}

Área de estudio

El estudio se realizó en la Parroquia El Tingo La Esperanza del Cantón Pujilí, ubicada en las coordenadas $0^{\circ} 54^{\prime} 52^{\prime \prime} \mathrm{S}, 79^{\circ} 03^{\prime} 26^{\prime \prime} \mathrm{O}$, a $1442 \mathrm{msnm}$, con una superficie aproximada de $205 \mathrm{~km}^{2}$, ubicada en la cordillera occidental de los Andes centrales del Ecuador (Figura 1). Conformada por 17 núcleos poblacionales con 844 UPA.

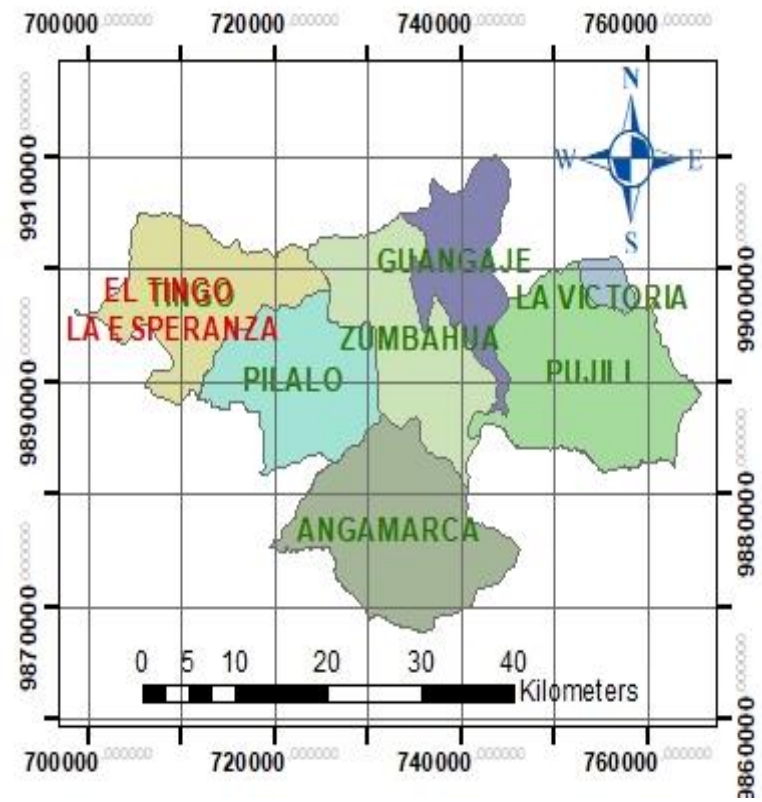

Figura 1. Ubicación geográfica de la parroquia El Tingo La Esperanza en la cordillera occidental de los Andes centrales del Ecuador.

\section{Metodología}

Sarandón (2002) indica que se debe aplicar encuestas estructuradas, basadas en indicadores socioculturales, económicos y ecológicos en las fincas seleccionadas, el levantamiento de esta información se realiza en campo.

De las 844 UPA, se realizó un muestreo simple estratificado en forma aleatoria. Para establecer la cantidad de encuestas se aplicó la Fórmula 1, de acuerdo a Scheaffer \& Mendenhall (1987).

$$
\mathrm{n}=\frac{\frac{4 \mathrm{PQ}}{\mathrm{d}^{2}}}{\frac{4 \mathrm{PQ}}{\mathrm{d}^{2}}-1}
$$

Fórmula 1. Algoritmo para establecer la cantidad de encuestas a aplicar (Scheaffer \& Mendenhall, 1987).

Donde:

$\mathrm{n}=$ Tamaño de la muestra (90)

$\mathrm{N}=$ Población Objetivo (844 UPA)

$\mathrm{P}=$ Probabilidad de acierto 0.5

$\mathrm{Q}=$ Probabilidad de error 0.5

$\mathrm{d}=$ Porcentaje de error

De los resultados de la muestra se derivaron 90 encuestas que fueron aplicadas a los principales núcleos poblacionales y se realizó un censo de la zona del proyecto de los efectos de la deforestación sobre la entomofauna rastrera de la microcuenca del río Yungañán, dando un total de 109 encuestas, para el estudio de la caracterización (Tabla 1).

Tabla 1. Asentamientos humanos de mayor importancia de la Parroquia El Tingo La Esperanza.

\begin{tabular}{lcc}
\hline Asentamientos & $\begin{array}{c}\text { Número de } \\
\text { UPA }\end{array}$ & $\begin{array}{c}\text { Muestra de } \\
\text { UPA }\end{array}$ \\
\hline Siete Ríos & 26 & 6 \\
Macuchi & 100 & 22 \\
El Tingo & 132 & 29 \\
El Palmar & $-\quad 98$ & 21 \\
Puembo & & \\
Choasilli & 71 & 16 \\
Yungañan & 15 & 15 \\
& Total encuestas: & 109 \\
\hline
\end{tabular}

Análisis de datos

Para el procesamiento y análisis de la información se aplicó el software estadístico InfoStat/L, versión 2019, el cual permite obtener cálculos de los coeficientes de variación (CV) de las 43 variables analizadas para discriminar aquellas que no contribuyen al análisis multivariado como lo recomienda Lores et al. (2008).

Se realizó el cálculo de las matrices de correlaciones con el objeto de identificar las variables fuertemente vinculadas entre sí para realizar la reducción dimensional con el uso del análisis de componentes principales (ACP), donde se identificaron las variables que influyen sobre la conformación de grupos, para luego realizar el análisis de conglomerados utilizando el método de Ward, tomando como medida la distancia métrica euclidiana graficada en un dendrograma (Guapi et al., 2017).

En el presente estudio para el análisis multivariado se siguieron las siguientes etapas: selección de la 


\section{E. JÁCOME, A. RODRÍGUEZ, S. JIMÉNEZ, K. MARÍN Y V. MOGRO}

Ecol. apl. Vol. $19 \mathrm{~N}^{\circ} 2$, pp. 49-56

muestra, aplicación de encuestas, tabulación de datos, análisis de variables para reducción dimensional (ACP) y agrupación de los sistemas agropecuarios más representativos del sector.

\section{Resultados y discusión}

Características socioeconómicas del productor

La responsabilidad del manejo de las UPA está a cargo en un $55.05 \%$ de mujeres (Tabla 2). La mayoría de los responsables se ubican en un rango de edad de 41 a 50 años con el $29.36 \%$, al igual que el rango de 51 a 60 años, que también representa el $29.36 \%$ del total de la muestra; las personas con edades entre 31 a 40 años representan el $20.18 \%$, mientras el $1.83 \%$ de los responsables forman parte del grupo de la tercera edad. Referente al nivel de instrucción de los responsables de las UPA, el $15.60 \%$ no posee ningún nivel académico, el $25.69 \%$ ha recibido instrucción primaria, el $44.95 \%$ secundaria y el $2.75 \%$ estudios universitarios.

Con respecto a la economía del hogar y las UPA, el $19.27 \%$ de las personas en estudio aportan solas, el $39.45 \%$ tiene la ayuda de una persona que contribuye con la economía, le siguen con $33.03 \%$ aquellos que tienen la ayuda de dos personas colaborando directamente para cubrir los gastos del hogar y las UPA, donde los ingresos mensuales del $49.54 \%$ bordea de 151 a 385 dólares, seguido del $15.60 \%$ que perciben un ingreso de 386 a 600 dólares y el $4.59 \%$ presenta ingresos de no más de 30 dólares, ubicándolos en un grupo de pobreza extrema.

El indicador de atención médica muestra que el $56.88 \%$ tiene la facilidad de contar con un centro de salud cercano a las UPA. Las características de la vivienda de las personas en estudio son muy básicas, pues el $45.87 \%$ solo posee un refugio, seguido de un $32.11 \%$ con casas de madera, y solo el $6.42 \%$ posee una casa adecuada. El medio de comunicación más utilizado es la radio con el $25.69 \%$ de la población, el $6.42 \%$ en el sector no posee medios de comunicación. El $9.17 \%$ no posee transporte público para poder llegar a sus hogares. El $76.15 \%$ de los habitantes se dedican a la agricultura, el $1.83 \%$ al comercio y el $6.42 \%$ a la ganadería. El $94.50 \%$ de los productores no han recibido capacitación, mientras que el $4.59 \%$ capacitado informa que el gobierno Provincial es la institución que más brinda apoyo en el sector, junto con el $0.92 \%$ que capacita la Universidad Técnica de Cotopaxi.

Características socioeconómicas de las unidades productivas agropecuarias (UPA)

En la Tabla 3 se indica que el $73.39 \%$ posee título de propiedad. El $50.46 \%$ posee una superficie de cultivo de 10000 a $50000 \mathrm{~m}^{2}$, seguido del $23.85 \%$ que posee una extensión menor a $1000 \mathrm{~m}^{2}$. El mayor porcentaje de las UPA, no tienen personas del núcleo familiar que ayuden en las labores de la finca con un $82.57 \%$. Los productores cuantifican su producción en quintales (bultos de $50 \mathrm{~kg}$ ), en un $39.45 \%$. La percepción de una producción buena es de $84.40 \%$. El $16.51 \%$ usa fertilizantes y solo un $4.59 \%$ utiliza semilla certificada.

El $32.11 \%$ realizan la comercialización en finca con intermediarios. El $21.10 \%$ definen a la calidad del producto por el tamaño y el $15.60 \%$ por la cantidad. Sumando el número de jornaleros el $61.46 \%$ de las UPA, contratan mano de obra externa de forma ocasional. El $23.85 \%$ de las fincas poseen un jornalero. Para la producción agrícola el $68.81 \%$ son dueños de las UPA. El $28.44 \%$ de los productores se siente conforme con su sistema de producción.

Factores ambientales del predio

En la Tabla 4 se observa que el $39.45 \%$ de los productores se definen como agricultores orgánicos, el $44.95 \%$ manifiesta realizar rotaciones de cultivo. El $62.39 \%$ espera las lluvias para el abastecimiento de agua en los cultivos. El $32.11 \%$ y el $15.60 \%$ manifiestan perdidas en sus cultivos por problemas de plagas y enfermedades respectivamente. El 19.27\% manifiesta realizar actividades de conservación del ambiente.

Los problemas citados anteriormente en la Tabla 4, se deben a que muchos productores del sector no aplican tecnología que les permita mejorar su productividad agropecuaria, la mayor proporción de habitantes se dedica a sembrar pastos para engorde de ganado vacuno, luego al monocultivo de caña de azúcar (Saccharum officinarum L.) y de mora (Rubus glaucus B.).

Tipos de unidades productivas agropecuarias (UPA) de la Parroquia El Tingo La Esperanza.

Del análisis multivariado ubicado en la Figura 2, en función de los componentes principales, en concordancia con Cáceres (2018), se determinó la relación entre variables de mayor importancia en el sistema de fincas agropecuarias del sector, donde el ingreso mensual de las familias tiene una relación directa con el tipo de vivienda, acceso a servicios básicos, acceso a la salud y disponibilidad de transporte como las variables que indican una mejor calidad de vida entre los productores, para luego realizar el agrupamiento de las UPA en función de sus similitudes mediante el análisis de conglomerados indicado en la Figura 3, donde se observan dos agrupamientos a una distancia ubicada en la mediana con un valor de 1.41; con estos resultados se determinó que los asentamientos humanos con mayor dificultad en su situación socioeconómica son: Yungañan y Siete Ríos, debido a la falta de servicios básicos, tipo de vivienda, bajos ingresos y características topográficas de los predios. El otro agrupamiento estuvo conformado por Macuchi, La Esperanza, El Palmar, Puembo y Choasilli. 


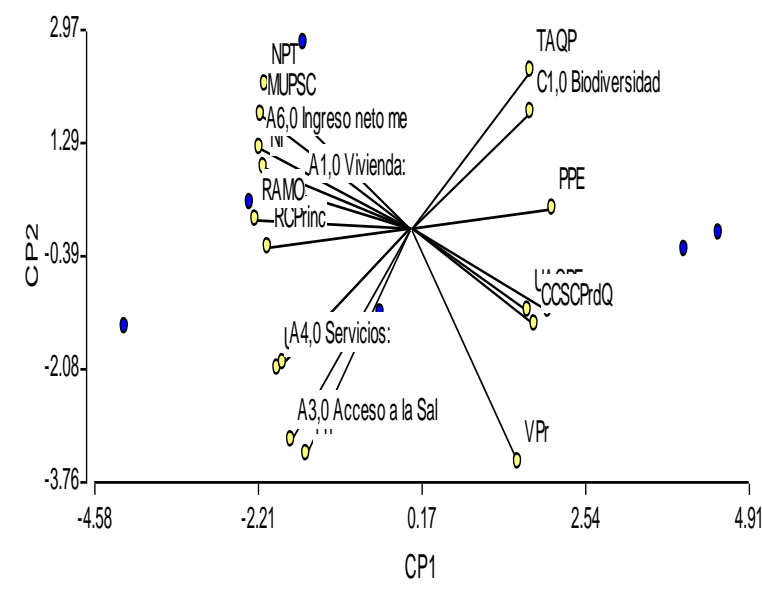

Figura 2. Análisis multivariado de los componentes principales de la caracterización de las unidades productivas agropecuarias (UPA) de la parroquia El Tingo La Esperanza.

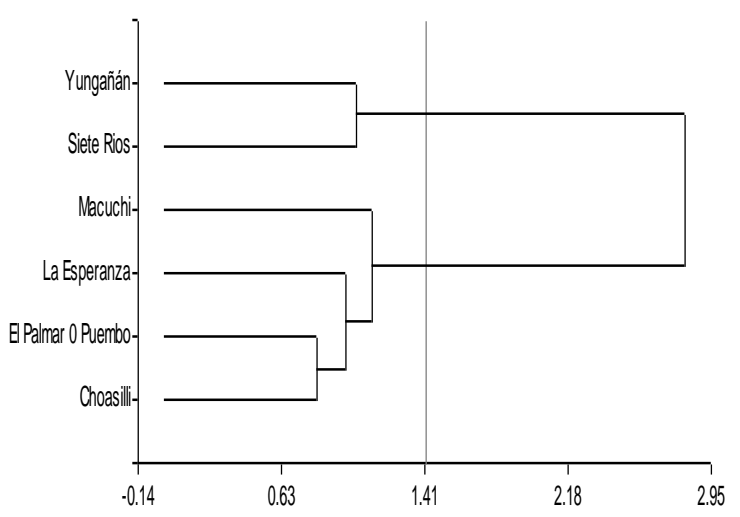

Figura 3. Análisis de conglomerados de la caracterización de las unidades productivas agropecuarias (UPA) de la parroquia El Tingo La Esperanza.

\section{Conclusiones}

Con el uso de 43 variables se caracterizaron los sistemas agropecuarios, los cuales son complejos, debido a que las familias en su mayoría se dedican al engorde de ganado vacuno, el cultivo de caña y el cultivo de mora; con escazas áreas destinadas a otros cultivos en los cuales se utiliza poca tecnología y cuya producción es destinada al autoconsumo. También se encontró un déficit importante de servicios básicos en la zona de estudio. El resultado del análisis de componentes principales indicó que el ingreso familiar está directamente relacionado con el acceso a servicios, para el agrupamiento con el uso de conglomerados se observan dos grupos de productores en función de sus similitudes, siendo los habitantes más vulnerables por la falta de vías de comunicación aquellos que están en las localidades de Siete Ríos y Yungañan. El otro agrupamiento estuvo conformado por Macuchi, El Tingo La Esperanza, El Palmar, Puembo y Choasilli.

$\mathrm{Se}$ recomienda conformar asociaciones de productores agropecuarios en el sector para mejorar las vías de comercialización, generando un valor agregado para mejorar los precios de los productos de la parroquia El Tingo La Esperanza.

\section{Agradecimientos}

Agradecimiento especial al $\mathrm{PhD}$ Carlos Torres Miño Director de Investigación de la Universidad Técnica de Cotopaxi y a los estudiantes de pregrado de la Carrera de Agronomía de la Universidad Técnica de Cotopaxi por la participación en las entrevistas, socialización y ejecución de las encuestas a los productores.

\section{Literatura citada}

Bolaños O. 1999. Caracterización y tipificación de organizaciones de productores y productoras. En: Unidad de planificación estratégica. Ministerio de agricultura y ganadería. 31-39. XI Congreso Nacional Agronómico/I Congreso Nacional de Extensión. San Jose, Costa Rica. http://www.mag.go.cr/congreso_agronomico_xi/a506907-I_031.pdf.

Cáceres H. 2018. Caracterización y tipología de fincas productoras de vid para Pisco en la región Ica-Perú. Idesia, 36(3): 35-43. https://scielo.conicyt.cl/scielo.php?pid=S071834292018005001002\&script=sci_arttext.

Guapi G., Masquiza M. \& Curbelo R. 2017. Caracterización de sistemas productivos lecheros en condiciones de montaña, Parroquia Químiag, Provincia Chimborazo, Ecuador. Revista de Producción Animal, 29(2): 14-17. https://revistas.reduc.edu.cu/index.php/rpa/article/view/1 684.

Lores A., Leyva A. \& Varela M. 2008. Los Dominios de Recomendaciones: Establecimiento e importancia para el análisis científico de los agroecosistemas. Cultrop, 29(3): 5-10. DOI: http://dx.doi.org/10.1234/ct.v29i3.236.

Ramírez B., Lavelle P., Orjuela J. \& Villanueva O. 2012. Caracterización de fincas ganaderas y adopción de sistemas agroforestales como propuesta de manejo de suelos en Caquetá, Colombia. Revista Colombiana de Ciencias Pecuarias, 25(3): 391-401. https://www.redalyc.org/comocitar.oa? $\mathrm{id}=29502492300$ 6.

Sarandón S. 2002. Evaluación de la sustentabilidad en agroecosistemas. Agroecología, 4: 19-28. https://wp.ufpel.edu.br/consagro/files/2010/10/SARAN DON-cap-20-Sustentabilidad.pdf.

Sarandón S., Zuluaga M., Cieza R., Gómez C., Janjetic L. \& Negrete E. 2006. Evaluación de la sustentabilidad de sistemas agrícolas de fincas en misiones, argentina, mediante el uso de indicadores. Cerealicultura. La plata, Argentina. Agroecología, 1: 19-28. https://scihub.wikicn.top/.

Scheaffer R. \& Mendenhall W. 1987. Elementos de muestreo. (G. R. A., Trad.) México D.F.: Grupo Editorial Iberoamericana S.A. 
Zayas R. 2013. Problematica municipal sustentabilidad y educacion agropopecuaria. Revista Mexicana de Agronegocios, $\quad 7(12)$ : 566-571. https://www.redalyc.org/pdf/141/14101205.pdf.

Tabla 2. Caracterización socioeconómica de los productores agropecuarios de la Parroquia El Tingo La Esperanza.

\begin{tabular}{|c|c|c|c|}
\hline Variable & Respuesta & Frecuencia & Porcentaje \\
\hline \multirow[t]{2}{*}{ 1.- Sexo del responsable de la Unidad de Producción } & Hombre & 49 & 44.95 \\
\hline & Mujer & 60 & 55.05 \\
\hline \multicolumn{4}{|l|}{ 2.- Edad entre la que se encuentra el responsable UPA } \\
\hline \multirow[t]{6}{*}{ (años) } & Ninguno & 1 & 0.92 \\
\hline & $20-30$ & 20 & 18.35 \\
\hline & $31-40$ & 22 & 20.18 \\
\hline & $41-50$ & 32 & 29.36 \\
\hline & $51-60$ & 32 & 29.36 \\
\hline & Más de 70 & 2 & 1.83 \\
\hline \multirow[t]{6}{*}{ 3.- Nivel de instrucción del responsable de la UPA } & Ninguno & 17 & 15.60 \\
\hline & Primaria (Inicial) & 28 & 25.69 \\
\hline & Secundaria (Bachillerato) & 49 & 44.95 \\
\hline & Técnico & 11 & 10.09 \\
\hline & Universitario & 3 & 2.75 \\
\hline & Posgrado & 1 & 0.92 \\
\hline \multirow[t]{6}{*}{ 4.- Número de hijos menores de 18 años } & 0 Hijos & 40 & 36.70 \\
\hline & 1 Hijo & 34 & 31.19 \\
\hline & 2 Hijos & 28 & 25.69 \\
\hline & 3 Hijos & 2 & 1.83 \\
\hline & 4 Hijos & 4 & 3.67 \\
\hline & 5 Hijos & 1 & 0.92 \\
\hline \multicolumn{4}{|l|}{ 5.- Número de personas que aportan con los gastos de } \\
\hline \multirow[t]{6}{*}{ la casa } & 0 Personas & 21 & 19.27 \\
\hline & 1 Persona & 43 & 39.45 \\
\hline & 2 Personas & 36 & 33.03 \\
\hline & 3 Personas & 5 & 4.59 \\
\hline & 5 Personas & 3 & 2.75 \\
\hline & 9 Personas & 1 & 0.92 \\
\hline \multirow[t]{3}{*}{ 6.- ¿Hay centro médico en su sector? } & Ninguno & 3 & 2.75 \\
\hline & No & 44 & 40.37 \\
\hline & $\mathrm{Si}$ & 62 & 56.88 \\
\hline \multirow[t]{6}{*}{ 7.- ¿Distancia próxima del centro médico? } & Ninguno & 9 & 8.26 \\
\hline & A 10 minutos & 36 & 33.03 \\
\hline & A 120 minutos & 12 & 11.01 \\
\hline & A 30 minutos & 33 & 30.28 \\
\hline & A 60 minutos & 4 & 3.67 \\
\hline & Acceso difícil & 15 & 13.76 \\
\hline \multirow[t]{5}{*}{ 8.- Vivienda } & Casa básica (refugio) & 50 & 45.87 \\
\hline & Casa de caña & 16 & 14.68 \\
\hline & Casa de madera & 35 & 32.11 \\
\hline & Casa mixta & 7 & 6.42 \\
\hline & No posee & 1 & 0.92 \\
\hline \multirow[t]{4}{*}{ 9.- Ingreso mensual en dólares } & $0-30$ & 5 & 4.59 \\
\hline & $31-150$ & 33 & 30.28 \\
\hline & $151-385$ & 54 & 49.54 \\
\hline & $386-600$ & 17 & 15.60 \\
\hline \multirow[t]{6}{*}{ 10.- ¿Cría animales? } & Otros & 19 & 17.43 \\
\hline & Bovinos & 18 & 16.51 \\
\hline & Ovinos & 12 & 11.01 \\
\hline & Cerdos & 23 & 21.10 \\
\hline & Cobayos & 6 & 5.50 \\
\hline & Aves & 31 & 28.44 \\
\hline \multirow{4}{*}{$\begin{array}{l}\text { 11.- Medio de comunicación e información que suele } \\
\text { utilizar }\end{array}$} & & & \\
\hline & Ninguno & 7 & 6.42 \\
\hline & Periódico & 17 & 15.60 \\
\hline & Radio & 28 & 25.69 \\
\hline
\end{tabular}


CARACTERIZACIÓN DE FINCAS AGROPECUARIAS DE EL TINGO LA ESPERANZA / ECUADOR Agosto - Diciembre 2020

\begin{tabular}{|c|c|c|c|}
\hline Variable & Respuesta & Frecuencia & Porcentaje \\
\hline \multirow{8}{*}{ 12. Transporte público } & Teléfono/celular & 26 & 23.85 \\
\hline & Internet & 2 & 1.83 \\
\hline & Más de tres medios & 29 & 26.61 \\
\hline & No cuenta & 10 & 9.17 \\
\hline & Transporte en la mañana, en & & \\
\hline & la tarde y en la noche. & 3 & 2.75 \\
\hline & Transporte solo de alquiler & 40 & 36.70 \\
\hline & Trasporte en la mañana y en & & \\
\hline \multirow{12}{*}{ 13.- Actividad a la que se dedica la Familia } & la tarde & 48 & 44.04 \\
\hline & Lunes a Viernes cada Hora & 8 & 7.37 \\
\hline & Lunes a Domingo cada & & \\
\hline & Hora & 0 & 0.00 \\
\hline & No realiza & 8 & 7.34 \\
\hline & Agricultura & 83 & 76.15 \\
\hline & Ganadería & 7 & 6.42 \\
\hline & Comercialización & 2 & 1.83 \\
\hline & Agricultura. Ganadería & 6 & 5.50 \\
\hline & Agricultura. & & \\
\hline & Comercialización & 2 & 1.83 \\
\hline & Ganadería. & & \\
\hline \multirow{3}{*}{ 14.- ¿Ha recibido capacitación por alguna entidad? } & Comercialización & 1 & 0.92 \\
\hline & No & 103 & 94.50 \\
\hline & $\mathrm{Si}$ & 6 & 5.50 \\
\hline \multirow[t]{3}{*}{ 15. ¿Cuál es la organización que le ha capacitado? } & Ninguna & 103 & 94.50 \\
\hline & GAD provincial & 5 & 4.59 \\
\hline & Universidad & 1 & 0.92 \\
\hline
\end{tabular}

UPA: Unidades Productivas Agropecuarias.

Tabla 3. Características socioeconómicas de las unidades productivas agropecuarias (UPA).

\begin{tabular}{|c|c|c|c|}
\hline Variable & Respuesta & Frecuencia & Porcentaje \\
\hline \multirow[t]{3}{*}{ 16.- ¿Tiene título de propiedad? } & Ninguno & 1 & 0.92 \\
\hline & No & 28 & 25.69 \\
\hline & $\mathrm{Si}$ & 80 & 73.39 \\
\hline \multirow{7}{*}{$\begin{array}{l}\text { 17.- Extensión de terreno de cultivo que posee }\left(\mathrm{m}^{2} \text {, }\right. \\
\text { hectáreas) }\end{array}$} & & & \\
\hline & De 1 a 20 hectareas & $\begin{array}{r}2 \\
55\end{array}$ & 1.83 \\
\hline & De 1 a 5 hectáreas & 55 & 50.46 \\
\hline & De 5 a 10 hectáreas & 3 & 2.75 \\
\hline & Más de 20 hectáreas & 5 & 4.59 \\
\hline & Menos de 1 hectárea & 18 & 16.51 \\
\hline & Menos de $1000 \mathrm{~m}^{2}$ & 26 & 23.85 \\
\hline \multirow[t]{4}{*}{$\begin{array}{l}\text { 18.- ¿Cuántas personas trabajan en su predio? } \\
\text { (incluido usted) }\end{array}$} & 0 Personas & 90 & 82.57 \\
\hline & 1 Persona & 3 & 2.75 \\
\hline & 2 Personas & 11 & 10.09 \\
\hline & 3 Personas & 5 & 4.59 \\
\hline \multirow[t]{4}{*}{$\begin{array}{l}\text { 19.- Capacidad de producción de los cultivos } \\
\text { agrícolas }\end{array}$} & Ninguno & 4 & 3.67 \\
\hline & $\mathrm{Kg} / \mathrm{ha}$ & 43 & 39.45 \\
\hline & Quintales & 43 & 39.45 \\
\hline & Sin producción & 19 & 17.43 \\
\hline \multirow{4}{*}{$\begin{array}{l}\text { 20.- ¿El rendimiento de su cultivo principal es? } \\
\text { (Si posee) }\end{array}$} & Ninguno & 10 & 9.17 \\
\hline & Bueno & 92 & 84.40 \\
\hline & Excelente & 4 & 3.67 \\
\hline & Malo & 3 & 2.75 \\
\hline \multirow[t]{8}{*}{ 21.- Para producir usa } & Otros & 37 & 33.94 \\
\hline & Semilla certificada & 5 & 4.59 \\
\hline & Fertilizantes & 18 & 16.51 \\
\hline & Insecticidas & 4 & 3.67 \\
\hline & Plantas injertadas & 5 & 4.59 \\
\hline & Almácigos & 8 & 7.34 \\
\hline & Compost & 30 & 27.52 \\
\hline & Fungicidas & 2 & 1.83 \\
\hline
\end{tabular}




\begin{tabular}{|c|c|c|c|}
\hline Variable & Respuesta & Frecuencia & Porcentaje \\
\hline \multirow[t]{8}{*}{$\begin{array}{l}\text { 22.- ¿Dónde vende los productos que obtiene en su } \\
\text { unidad de producción? }\end{array}$} & Ninguno & 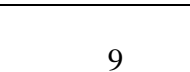 & 8.26 \\
\hline & Asociación de productores & 8 & 7.34 \\
\hline & Intermediarios & 35 & 32.11 \\
\hline & Local e Intermediarios & 45 & 41.28 \\
\hline & Mercado Local & 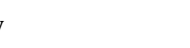 & \\
\hline & Asociación & 6 & 5.50 \\
\hline & Mercado Mayorista & & \\
\hline & Asocia & 6 & 5.50 \\
\hline \multirow[t]{5}{*}{ 23.- La calidad de su producto lo define por } & Ninguno & 46 & 42.20 \\
\hline & Tamaño & 23 & 21.10 \\
\hline & Color & 6 & 5.50 \\
\hline & Forma & 17 & 15.60 \\
\hline & Cantidad & 17 & 15.60 \\
\hline \multirow[t]{3}{*}{ 24.- ¿Utiliza peones o mano de obra contratada? } & Ninguno & 7 & 6.42 \\
\hline & No & 72 & 66.06 \\
\hline & $\mathrm{Si}$ & 30 & 27.52 \\
\hline \multirow{8}{*}{$\begin{array}{l}\text { 25.- Número de Jornaleros que trabajan (incluido } \\
\text { usted) }\end{array}$} & & & \\
\hline & 0 Jornaleros & 42 & 38.53 \\
\hline & 1 Jornalero & 26 & 23.85 \\
\hline & 2 Jornaleros & 19 & 17.43 \\
\hline & 3 Jornaleros & 18 & 16.51 \\
\hline & 4 Jornaleros & 2 & 1.83 \\
\hline & 5 Jornaleros & 1 & 0.92 \\
\hline & 6 Jornaleros & 1 & 0.92 \\
\hline \multirow[t]{6}{*}{ 26.- ¿Cuál es el costo de un jornal? } & 0 USD & 55 & 50.46 \\
\hline & 10 USD & 10 & 9.17 \\
\hline & 12 USD & 4 & 3.67 \\
\hline & 15 USD & 38 & 34.86 \\
\hline & 15 a 20 USD & 1 & 0.92 \\
\hline & Más de 20 USD & 1 & 0.92 \\
\hline \multirow[t]{4}{*}{ 27.- Tenencia de la tierra (si posee) } & Ninguno & 25 & 22.94 \\
\hline & Herencia & 1 & 0.92 \\
\hline & No posee & 8 & 7.34 \\
\hline & Posee título & 75 & 68.81 \\
\hline \multirow[t]{7}{*}{ 28.- ¿Cómo se siente con la actividad que realiza? } & Ninguno & 8 & 7.34 \\
\hline & $\begin{array}{l}\text { No se siente del todo } \\
\text { satisfecho }\end{array}$ & 31 & 28.44 \\
\hline & Poco satisfecho & 6 & 5.50 \\
\hline & Se siente desilusionado & 1 & 0.92 \\
\hline & Se siente feliz & 18 & 16.51 \\
\hline & Se siente muy feliz & 14 & 12.84 \\
\hline & Se siente satisfecho & 31 & 28.44 \\
\hline
\end{tabular}

Tabla 4. Características ambientales de las unidades productivas agropecuarias (UPA).

\begin{tabular}{|c|c|c|c|}
\hline Variable & Respuesta & Frecuencia & Porcentaje \\
\hline \multirow[t]{3}{*}{$\begin{array}{l}\text { 29.- ¿Cuenta con agua de riego permanente durante } \\
\text { todo el año? }\end{array}$} & Ninguno & 2 & 1.83 \\
\hline & No & 81 & 74.31 \\
\hline & $\mathrm{Si}$ & 26 & 23.85 \\
\hline \multirow[t]{4}{*}{ 30.- ¿Cuál es la fuente de abastecimiento del agua? } & Lluvia & 68 & 62.39 \\
\hline & Pozo & 11 & 10.09 \\
\hline & Río & 14 & 12.84 \\
\hline & Canal de riego & 16 & 14.68 \\
\hline \multirow[t]{3}{*}{ 31.- ¿Qué tipo de agricultura posee? } & Ninguno & 4 & 3.67 \\
\hline & Convencional & 62 & 56.88 \\
\hline & Orgánica & 43 & 39.45 \\
\hline \multirow[t]{3}{*}{ 32.- ¿Utiliza abono químico para la fertilización? } & Ninguno & 3 & 2.75 \\
\hline & No & 81 & 74.31 \\
\hline & $\mathrm{Si}$ & 25 & 22.94 \\
\hline
\end{tabular}


CARACTERIZACIÓN DE FINCAS AGROPECUARIAS DE EL TINGO LA ESPERANZA / ECUADOR Agosto - Diciembre 2020

\begin{tabular}{|c|c|c|c|}
\hline Variable & Respuesta & Frecuencia & Porcentaje \\
\hline \multicolumn{4}{|c|}{ 33.- ¿Controla los cultivos solo con productos } \\
\hline \multirow[t]{2}{*}{ químicos? } & Ninguno & $\begin{array}{r}3 \\
01\end{array}$ & $\begin{array}{r}2.75 \\
83.49\end{array}$ \\
\hline & $\mathrm{Si}$ & 15 & 13.76 \\
\hline \multirow{4}{*}{$\begin{array}{l}\text { 34.- ¿Mantiene su unidad de producción siempre } \\
\text { cubierta? }\end{array}$} & & & \\
\hline & Ninguno & 3 & 2.75 \\
\hline & No & 32 & 29.36 \\
\hline & $\mathrm{Si}$ & 74 & 67.89 \\
\hline \multirow[t]{3}{*}{ 35.- ¿Realiza quema de rastrojo de maleza? } & Ninguno & 3 & 2.75 \\
\hline & No & 78 & 71.56 \\
\hline & $\mathrm{Si}$ & 28 & 25.69 \\
\hline \multirow[t]{3}{*}{ 36.- ¿Realiza aplicación de materia orgánica? } & Ninguno & 4 & 3.67 \\
\hline & No & 19 & 17.43 \\
\hline & $\mathrm{Si}$ & 86 & 78.90 \\
\hline \multirow[t]{3}{*}{ 37.- ¿Realiza rotación de cultivo? } & Ninguno & 3 & 2.75 \\
\hline & No & 57 & 52.29 \\
\hline & $\mathrm{Si}$ & 49 & 44.95 \\
\hline \multirow[t]{5}{*}{ 38.- ¿Cada qué tiempo rota los cultivos? } & Ninguno & 7 & 6.42 \\
\hline & Cada 2 años & 6 & 5.50 \\
\hline & Cada 3 años o mas & 5 & 4.59 \\
\hline & Cada año & 34 & 31.19 \\
\hline & No realiza & 57 & 52.29 \\
\hline \multirow{4}{*}{$\begin{array}{l}\text { 39.- ¿Utiliza repelente o extracto para combatir plagas } \\
\text { hechas por usted? }\end{array}$} & & & \\
\hline & Ninguno & 3 & 2.75 \\
\hline & No & 98 & 89.91 \\
\hline & $\mathrm{Si}$ & 8 & 7.34 \\
\hline \multirow{3}{*}{ 40.- ¿Realiza controles biológicos en sus cultivos? } & Ninguno & 3 & 2.75 \\
\hline & No & 100 & 91.74 \\
\hline & $\mathrm{Si}$ & 6 & 5.50 \\
\hline \multirow[t]{9}{*}{$\begin{array}{l}\text { 41.- ¿Cuál es el problema de mayor incidencia } \\
\text { durante los cultivos? }\end{array}$} & Otros & 9 & 8.26 \\
\hline & Plagas & 35 & 32.11 \\
\hline & Enfermedades & 17 & 15.60 \\
\hline & Malezas & 25 & 22.94 \\
\hline & Insuficiencias de abonos & 2 & 1.83 \\
\hline & Sequias & 6 & 5.50 \\
\hline & Plagas. Enfermedades & 3 & 2.75 \\
\hline & Pagas. Malezas & 10 & 9.17 \\
\hline & $\begin{array}{l}\text { Pagas. Insuficiencias de } \\
\text { abonos }\end{array}$ & 2 & 1.83 \\
\hline \multirow[t]{3}{*}{$\begin{array}{l}\text { 42.- ¿Posee pendiente de erosión en su unidad de } \\
\text { producción? }\end{array}$} & Ninguno & 3 & 2.75 \\
\hline & No & 69 & 63.30 \\
\hline & $\mathrm{Si}$ & 37 & 33.94 \\
\hline \multirow{4}{*}{$\begin{array}{l}\text { 43.- ¿Realiza obras de conservación de suelos como } \\
\text { terrazas, zanjas de desviación, canterones, intercalado } \\
\text { de especies forestales o cortinas rompevientos? }\end{array}$} & & & \\
\hline & Ninguno & 4 & 3.67 \\
\hline & No & 84 & 77.06 \\
\hline & $\mathrm{Si}$ & 21 & 19.27 \\
\hline
\end{tabular}

\footnotetext{
${ }^{1}$ Universidad Técnica de Cotopaxi, Facultad de Ciencias Agropecuarias y Recursos Naturales, Salache Bajo s/n, Latacunga, Ecuador.

2 emerson.jacome@utc.edu.ec.

${ }^{3}$ Universidad Nacional Agraria La Molina, Av. La Molina s/n, Lima 12, Perú. arodriber@lamolina.edu.pe.

${ }^{4}$ cristian.jimenez@utc.edu.ec.

${ }^{5}$ karina.marin@utc.edu.ec.

${ }^{6}$ vinicio.mogro@utc.edu.ec.
} 